\title{
Impact on disease-free survival of the duration of ovarian function suppression, as postoperative adjuvant therapy, in premenopausal women with hormone receptor-positive breast cancer: a retrospective single-institution study
}

\author{
Yukinori Ozaki ${ }^{1}$ - Yuko Tanabe ${ }^{1}$ Nobuko Tamura ${ }^{2} \cdot$ Takuya Ogura $^{2} \cdot$ Chihiro Kondoh $^{1} \cdot$ Yuji Miura $^{1}$. \\ Kenichi Yoshimura ${ }^{3} \cdot$ Hidetaka Kawabata $^{2} \cdot$ Toshimi Takano $^{1}$
}

Received: 11 October 2017 / Accepted: 17 January 2018 / Published online: 22 January 2018

(c) The Author(s) 2018. This article is an open access publication

\begin{abstract}
Introduction Although tamoxifen (TAM) plus ovarian function suppression (OFS) is considered as a standard adjuvant treatment for premenopausal women with hormone receptor-positive breast cancer, the optimal duration of OFS has not yet been established. This retrospective study was designed to assess the duration of OFS and the impact of the duration of OFS on the DFS in these patients.

Methods We retrospectively reviewed the data of premenopausal patients with breast cancer who received TAM + OFS (goserelin or leuprorelin) as adjuvant therapy between February 2004 and June 2015. The primary analysis was a comparison of the disease-free survival (DFS) between patients who received OFS for 3 years or less (OFS $\leq 3$ years group) and those who received OFS for longer than 3 years (OFS $>3$ years group).

Results We analyzed the data of 215 premenopausal patients diagnosed as having hormone receptor-positive breast cancer. A propensity score-matched model showed the absence of any significant difference in the DFS between the OFS $\leq 3$ years group and OFS > 3 years group (6-year DFS rate, 93.2 vs. 94.0\%; log-rank test $p=0.767$ ).

Conclusions Our data showed that among premenopausal women with hormone receptor-positive breast cancer who received TAM + OFS as adjuvant endocrine therapy, there was no significant difference in the DFS between the OFS $\leq 3$-year group and OFS $>3$-year group. A randomized trial is needed to establish the optimal duration of OFS for these patients.
\end{abstract}

Keywords Hormone receptor-positive · Breast cancer · Premenopausal · Ovarian function suppression · Duration · Propensity

Yukinori Ozaki

1755ozaki@toranomon.gr.jp

Yuko Tanabe

ytanabe@toranomon.gr.jp

Nobuko Tamura

nobtamura@toranomon.gr.jp

Takuya Ogura

ogutaku0120@gmail.com

Chihiro Kondoh

ckondoh@toranomon.gr.jp

Yuji Miura

yujmiura@mac.com

Kenichi Yoshimura

keyoshim@staff.kanazawa-u.ac.jp
Hidetaka Kawabata

h-kawabata@toranomon.gr.jp

Toshimi Takano

takano@toranomon.gr.jp

1 Department of Medical Oncology, Toranomon Hospital, 2-2-2 Toranomon, Minato-ku, Tokyo 105-8470, Japan

2 Department of Breast and Endocrine Surgery, Toranomon Hospital, 2-2-2 Toranomon, Minato-ku, Tokyo 105-8470, Japan

3 Innovative Clinical Research Center, Kanazawa University Hospital, Kakuma-city, Kanazawa, Ishikawa 920-8641, Japan 


$\begin{array}{ll}\text { Abbreviations } \\ \text { TAM } & \text { Tamoxifen } \\ \text { OFS } & \text { Ovarian function suppression } \\ \text { DFS } & \text { Disease-free survival } \\ \text { AJCC } & \text { American Joint Committee on Cancer } \\ \text { IHC } & \text { Immunohistochemistry } \\ \text { OS } & \text { Overall survival } \\ \text { FEC } & \text { Fluorouracil + epirubicin + cyclophosphamide } \\ \text { AC } & \text { Doxorubicin + cyclophosphamide } \\ \text { RR } & \text { Risk ratio } \\ \text { QOL } & \text { Quality of life }\end{array}$

\section{Introduction}

Tamoxifen (TAM) plus ovarian function suppression (OFS) is one of the standard adjuvant treatments for premenopausal women with hormone receptor-positive breast cancer. Although some prospective studies have suggested that adjuvant TAM plus OFS provides a disease-free survival (DFS) benefit as compared to TAM alone, there is insufficient evidence as to the optimal duration of OFS needed in patients receiving adjuvant OFS in combination with TAM [1-3].

The prognosis of premenopausal women with hormone receptor-positive breast cancer is worse than that of postmenopausal women with breast cancer $[4,5]$. The Suppression of Ovarian Function Trial (SOFT), a randomized phase III trial conducted by The International Breast Cancer Study Group (IBCSG), showed significant risk reduction, in terms of the DFS, obtained with the addition of OFS to TAM in women who were at a sufficient risk for recurrence to warrant adjuvant chemotherapy and who remained premenopausal until the end of the adjuvant chemotherapy; however, overall analysis of the patients in this trial failed to reveal significant benefit of the addition of OFS to TAM [6]. Therefore, the clinical benefit/additional toxicity balance of the addition of OFS to TAM should be carefully weighed.

The International Breast Cancer Study Group Trial VIIIA showed a significant benefit of the addition of OFS for 2 years to adjuvant chemotherapy in premenopausal women with lymph node-negative hormone receptor-positive breast cancer [7]. In the Austrian Breast and Colorectal Cancer Study Group Trial, while use of OFS for 3 years after adjuvant chemotherapy failed to show significant benefit in the overall patient population, a significant reduction in the risk of recurrence was reported in patients $<40$ years of age [8]. Several meta-analyses have suggested that addition of OFS to TAM improved the DFS as compared to TAM alone in premenopausal patients younger than 40 years old; the duration of OFS in these studies varied from 2 to 3 years [9]. Based on the results of the SOFT trial, the American Society of Clinical Oncology clinical practice guideline recommends OFS for duration of 5 years [10]. In one randomized controlled pilot study conducted to determine the optimal duration of OFS, the survival benefit obtained with adjuvant leuprorelin treatment was similar between premenopausal patients treated for longer than 3 years and those treated for 2 years [11]. However, there are few data on the impact of the duration of OFS as adjuvant endocrine therapy, and the optimal duration of OFS has not yet been clearly established.

We conducted a single-institutional retrospective study to compare the DFS between patients who had received OFS for 3 years or less and those who had received OFS for longer than 3 years as adjuvant therapy. A propensity score-matched model was used for the analysis to minimize the effects of confounding factors.

\section{Patients and methods}

\section{Patients}

This study was designed as a retrospective review of the medical records of premenopausal patients with breast cancer who had received TAM plus OFS (goserelin or leuprorelin) as adjuvant therapy between February 2004 and June 2015 at Toranomon Hospital. The key inclusion criteria were as follows: (1) premenopausal women who had been diagnosed as having invasive breast carcinoma, Stage I-III, according to the American Joint Committee on Cancer (AJCC) staging system, Seventh Edition [12]; (2) patients with regular menstrual cycles or premenopausal hormone levels after chemotherapy; (3) patients who had undergone partial mastectomy or mastectomy and sentinel lymph node biopsy and/or axillary lymph node dissection; (4) patients in whom tumor immunohistochemistry (IHC) showed positive results for ER and/or PgR; (5) patients who had received standard adjuvant endocrine therapy with tamoxifen plus goserelin or leuprorelin. This study did not include patients who received bilateral salpingo-oophorectomy, because it was rarely performed as OFS in our institution.

Approval for this retrospective study was obtained from the Ethics Committee of Toranomon Hospital.

Clinicopathologic data were collected from the medical records, including the age, ER, PgR, HER2 and Ki67 status, nuclear grade, clinical and pathological stage, details of the neo-adjuvant/adjuvant chemotherapy given, and duration of OFS and DFS. Patients were defined as ER- and/or PgRpositive when the tumor IHC showed an Allred score for ER and/or PgR of $\geq 2$. The tumor HER2 status was defined as positive when IHC for HER 2 showed a $3+$ or $2+$ score, with confirmation by fluorescence in situ hybridization. 


\section{Treatment}

All patients received adjuvant hormone therapy with oral TAM at the dose of $20 \mathrm{mg}$ daily for longer than 5 years. OFS was accomplished with a subcutaneous depot injection of goserelin acetate (Zoladex, AstraZeneca) at the dose of $3.6 \mathrm{mg}$ once every month or $10.8 \mathrm{mg}$ once every 3 months, or leuprorelin acetate (Leuplin, Takeda) at the dose of $3.75 \mathrm{mg}$ once every month or $11.25 \mathrm{mg}$ once every 3 months. The duration of TAM treatment and OFS was left to the discretion of the treating physician. The duration of OFS was calculated from the date of initiation to the date of completion of treatment with a luteinizing hormone-releasing hormone analogue or to a DFS event.

\section{Endpoints and statistical analysis}

The primary endpoint of the study was the DFS, defined as the length of survival time after primary treatment without any signs or symptoms of cancer, including loco-regional recurrence, contralateral breast cancer, distant recurrence, and second malignancy. Loco-regional recurrence was defined as pathologically confirmed disease recurrence in the ipsilateral chest wall, or within the supra-clavicular, subclavian, ipsilateral axillary, or ipsilateral internal mammary lymph nodes. The secondary endpoint was the overall survival (OS). The primary analysis was a comparison of the DFS between patients who had received OFS for 3 years or less (OFS $\leq 3$-year group) and those who had received OFS for longer than 3 years (OFS $>3$-year group), using the Cox proportional hazards model, propensity score-matched model. Because of the non-randomized design of this retrospective study, we used propensity score-matched models to reduce the influence of differences in the confounding factors between the OFS $\leq 3$ years and OFS $>3$-year groups. Propensity score was calculated based on the age, pT, pN, HER2 status, and perioperative chemotherapy. These data were analyzed using the JMP ver.12.0.1 (2015 SAS Institute Inc.). The Kaplan-Meier method was used for the survival analyses, and the log-rank test was used to compare the survivals between two groups, and univariate and multivariate analyses were conducted using the Cox proportional hazards model. Differences between the two groups were assessed by the Chi-square test. $p$ values less than 0.05 were considered to denote significance.

\section{Results}

The medical records of patients were searched from the database of our institution using the search terms "breast cancer" and "goserelin acetate" or "leuprorelin acetate" for the period between February 2004 and June 2015, and 532 patients were detected. Among these, 215 patients met the inclusion criteria for this study. The median follow-up duration of the patients was 71 months (range 20.3-144.3). The patient characteristics are shown in Table 1 . The median age was 42 years (range 24-53). The number of patients with pathological (p) T0-T1 was 149 (69\%), and that of patients with pN0 was 164 (76\%). The HER2 status was positive in $15(7 \%)$ patients. Neo-adjuvant or adjuvant chemotherapy had been administered in $66(31 \%)$ patients. The regimens used for the neo-adjuvant or adjuvant chemotherapy were fluorouracil + epirubicin + cyclophosphamide (FEC), conventional doxorubicin + cyclophosphamide (AC)

Table 1 Patient characteristics

\begin{tabular}{|c|c|c|}
\hline Characteristics & No. & $\%$ \\
\hline Age & $N=215$ & \\
\hline \multicolumn{3}{|c|}{ Median 42 years (range 24-53) } \\
\hline$\leq 40$ & 86 & 40 \\
\hline$>40$ & 129 & 60 \\
\hline \multicolumn{3}{|l|}{ pT stage } \\
\hline T0 & 1 & 0.5 \\
\hline Tis & 2 & 1 \\
\hline $\mathrm{T} 1$ & 146 & 68 \\
\hline $\mathrm{T} 2$ & 52 & 24 \\
\hline T3 & 12 & 6 \\
\hline $\mathrm{T} 4$ & 2 & 1 \\
\hline \multicolumn{3}{|l|}{$\mathrm{pN}$ stage } \\
\hline No & 164 & 76 \\
\hline N1 & 42 & 20 \\
\hline $\mathrm{N} 2$ & 8 & 4 \\
\hline $\mathrm{N} 3$ & 1 & 0.5 \\
\hline \multicolumn{3}{|l|}{$\mathrm{Ki}-67$} \\
\hline \multicolumn{3}{|c|}{ Median 8 (range $0-80)(\%)$} \\
\hline $0-20$ & 112 & 52 \\
\hline$>20$ & 20 & 9 \\
\hline Unknown & 83 & 39 \\
\hline \multicolumn{3}{|l|}{ HER 2 status } \\
\hline Positive & 15 & 7 \\
\hline Negative & 200 & 93 \\
\hline \multicolumn{3}{|l|}{ Nuclear grade } \\
\hline NG1 & 136 & 63 \\
\hline NG2 & 49 & 23 \\
\hline NG3 & 17 & 8 \\
\hline Unknown & 11 & 5 \\
\hline \multicolumn{3}{|c|}{ Neo-adjuvant/adjuvant chemotherapy } \\
\hline Yes & 66 & 31 \\
\hline No & 149 & 69 \\
\hline \multicolumn{3}{|c|}{ Duration of OFS } \\
\hline \multicolumn{3}{|c|}{ Median 896 (range 104-3676) (day) } \\
\hline$\leq 3$ years & 152 & 71 \\
\hline$>3$ years & 63 & 29 \\
\hline
\end{tabular}




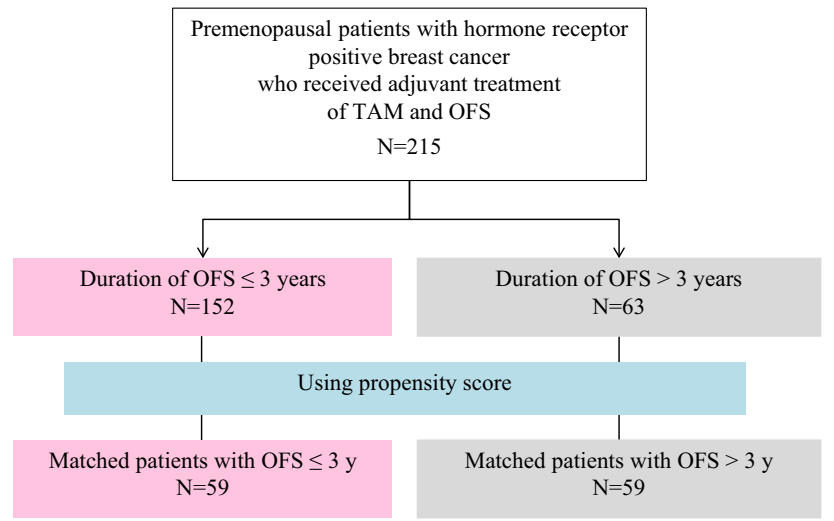

Fig. 1 Patient diagram. Based on the propensity score, the OFS $\leq 3$ years and OFS $>3$-year groups had 59 matched patients each that were included for the primary analysis

or dose-dense AC, followed by weekly paclitaxel or triweekly docetaxel. Trastuzumab was given for patients with an HER2-positive disease status. Of all the patients, 152 $(71 \%)$ received OFS for $\leq 3$ years and $63(29 \%)$ received OFS $>3$ years.

Propensity score matching was used to make the two groups with similar in terms of the baseline characteristics, based on the age, $\mathrm{pT}$ stage, $\mathrm{pN}$ stage, HER2 status, and the neo-adjuvant or adjuvant chemotherapy regimens used. The patient diagram is shown in Fig. 1. Based on the propensity score, the OFS $\leq 3$ years and OFS $>3$-year groups had 59 matched patients each that were included for the primary analysis. The patient characteristics in the two groups are shown in Table 2 . The age ( $\leq 40 />40$ years) $(p=0.852)$, pT $(\leq 2 \mathrm{~cm} />2 \mathrm{~cm})(p=0.853), \mathrm{pN}$ status (negative/positive) ( $p=0.849)$, HER2 status (negative/positive) $(p=0.507)$, nuclear grade $(p=0.922)$, and chemotherapy regimens employed $(p=0.577)$ were well balanced between the OFS $\leq 3$ years and OFS $\leq 3$-year groups.

The primary analysis showed the absence of any significant difference in the DFS between the OFS $\leq 3$ years and OFS > 3-year groups (hazard ratio 0.82, 95\% CI 0.21-2.89, log-rank test $p=0.767)$. The DFS rate at 6 years was $93.2 \%$ in the OFS $\leq 3$ years group and $94.0 \%$ in the OFS $>3$ years group (Fig. 2). The results of univariate Cox regression analysis conducted to identify factors influencing the DFS in the matched patients are shown in Table 3. The risk ratio (RR) of age $\leq 40$ and $\mathrm{pT}>2 \mathrm{~cm}$ was significantly greater, at 4.25 (95\% CI $1.15-20.30, p=0.029)$ and $>20(p<0.0001)$, respectively. Node-positive status (RR 1.21, 95\% CI 0.33-4.37), the chemotherapy regimen used (RR 2.19, 95\% CI 0.60-10.30), nuclear grade $>1$ (RR 1.13, 95\% CI 0.27-4.29), and HER2-positive status (RR 1.44, 95\% CI 0.07-7.87) were not identified as having any significant influence on the prognosis in either of the groups. Multivariate Cox regression analysis conducted to identify factors influencing the DFS in the matched patients
Table 2 Patient characteristics in the propensity score-matched groups

\begin{tabular}{|c|c|c|c|c|c|}
\hline \multirow[t]{2}{*}{ Characteristics } & \multicolumn{2}{|c|}{ OFS $\leq 3$ years } & \multicolumn{2}{|c|}{ OFS $>3$ years } & \multirow[t]{2}{*}{$p$} \\
\hline & $N=59$ & $(\%)$ & $N=59$ & $(\%)$ & \\
\hline \multicolumn{6}{|l|}{ Age } \\
\hline$\leq 40$ years & 25 & 42 & 26 & 44 & \\
\hline$>40$ years & 34 & 58 & 33 & 56 & 0.852 \\
\hline \multicolumn{6}{|l|}{ pT } \\
\hline$\leq 2 \mathrm{~cm}$ & 31 & 53 & 32 & 54 & \\
\hline$>2 \mathrm{~cm}$ & 28 & 47 & 27 & 46 & 0.853 \\
\hline \multicolumn{6}{|l|}{$\mathrm{pN}$} \\
\hline Negative & 36 & 61 & 37 & 63 & \\
\hline Positive & 23 & 39 & 22 & 37 & 0.849 \\
\hline \multicolumn{6}{|l|}{ HER 2 status } \\
\hline Positive & 4 & 7 & 6 & 10 & \\
\hline Negative & 55 & 93 & 53 & 90 & 0.507 \\
\hline \multicolumn{6}{|l|}{ Nuclear grade } \\
\hline NG1 & 33 & 56 & 36 & 61 & \\
\hline $\mathrm{NG} 2,3$ & 20 & 34 & 21 & 36 & 0.922 \\
\hline \multicolumn{6}{|c|}{ Neo-adjuvant/adjuvant chemotherapy } \\
\hline Yes & 35 & 59 & 32 & 54 & \\
\hline No & 24 & 41 & 27 & 46 & 0.577 \\
\hline
\end{tabular}

showed that OFS $\leq 3$ years was not a prognostic factor (RR $1.16,95 \%$ CI $0.29-4.18$ ), while age $\leq 40$ years (RR 4.01 , 95\% CI 1.08-19.31, $p=0.038)$ and $\mathrm{pT}>2 \mathrm{~cm}(\mathrm{RR}>20$, $p<0.0001$ ) were significant prognostic factors (Table 4).

For all the included patients, the Cox proportional hazards model using the duration of OFS and propensity score showed that the RR of OFS $\leq 3$ years was $0.82(p=0.750)$, which was not significantly different from the RR in the OFS $>3$-years group.

The 6-year DFS of the patients, overall, was 95.2\% (95\% CI 90.6-97.6), and the 10-year DFS was $84.0 \%$ (95\% CI 63.1-94.2) (Fig. 3). In patients who were younger than 40 years of age and/or had received perioperative chemotherapy, the 6-year DFS was 93.4\% (95\% CI 86.1-97.0). The RR of OFS $\leq 3$ years in these patients was $0.43(p=0.217)$, which was not significant.

A total of 13 DFS events were detected in the study population ( 6 events in the OFS $\leq 3$-year group, and 7 events in the OFS $>3$ years group), and 2 patients died of the breast cancer. OS as a secondary endpoint was not analyzed because of the short follow-up period and infrequent OS events.

\section{Discussion}

Analyses of the retrospective data using propensity score matching showed no statistically significant difference in the DFS between patients who had received adjuvant 
Fig. 2 Disease-free survival in propensity matched patients. Kaplan-Meier curve of DFS between the OFS $\leq 3$ years and OFS $>3$-year groups (hazard ratio $0.82,95 \%$ CI $0.21-2.89$, $\log$-rank test $p=0.767)$. The DFS rate at 6 years was $93.2 \%$ in the OFS $\leq 3$-year group and $94.0 \%$ in the OFS $>3$-year group

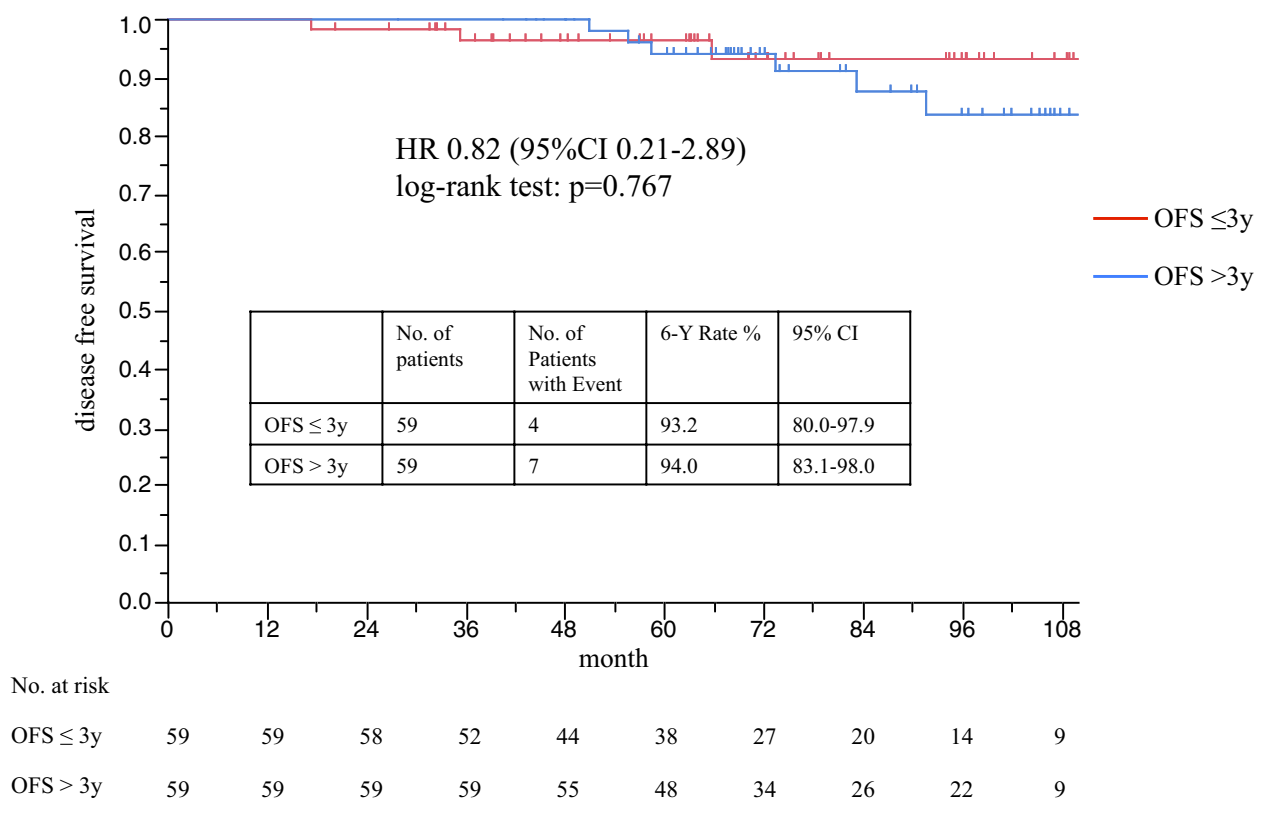

Table 3 Univariate cox regression analysis to identify factors influencing the DFS in propensity score-matched patients

\begin{tabular}{llcl}
\hline & Risk ratio & $p$ & $95 \%$ CI \\
\hline OFS $\leq 3$ years & 0.82 & 0.767 & $0.21-2.89$ \\
Age $\leq 40$ years & 4.25 & 0.029 & $1.15-20.30$ \\
pT $>2 \mathrm{~cm}$ & $>20$ & $<0.0001$ & NE \\
N-positive & 1.21 & 0.767 & $0.33-4.37$ \\
Chemotherapy & 2.19 & 0.238 & $0.60-10.30$ \\
Nuclear grade $>1$ & 1.13 & 0.858 & $0.27-4.29$ \\
HER2-positive & 1.44 & 0.741 & $0.07-7.87$ \\
\hline
\end{tabular}

Table 4 Multivariate cox regression to identify factors influencing the DFS in the matched patient groups

\begin{tabular}{llcl}
\hline & Risk ratio & $p$ & $95 \%$ CI \\
\hline OFS $\leq 3$ years & 1.16 & 0.822 & $0.29-4.18$ \\
Age $\leq$ 40 years & 4.01 & 0.038 & $1.08-19.31$ \\
pT $>2 \mathrm{~cm}$ & $>20$ & $<0.0001$ & NE \\
\hline
\end{tabular}

endocrine therapy plus OFS for $\leq 3$ years and those who had received it for $>3$ years. Although prospective studies have suggested a role of OFS in premenopausal women with hormone receptor-positive breast cancer, few studies have compared the clinical benefit of OFS for $\leq 3$ and $>3$ years. To the best of our knowledge, this is the first retrospective study to compare the impact on the DFS of OFS for $\leq 3$ years and OFS for $>3$ years administered in combination with TAM as adjuvant therapy in premenopausal breast cancer patients.
The duration of OFS had been left to the discretion of the attending physicians and the patients' wishes, depending on the biological and clinical features of the disease. Before publication of the SOFT trial, some patients received OFS for less than 3 years, although patients who were thought by their attending physicians as being at a high risk for recurrence received OFS for longer than 3 years at our institution. Propensity score matching was used to minimize confounding factor bias in this retrospective study. The DFS was not significantly different among the propensity score-matched model and Cox proportional hazards model, suggesting that OFS $\leq 3$ years was as effective as OFS $>3$ years in this population.

Adverse events, including hot flushes, depression, musculoskeletal symptoms, and quality of life (QOL) data, were not evaluated in this study. The health-related QOL data of the E-3193 trial showed that addition of OFS to TAM as adjuvant therapy resulted in a more significant increase of the menopausal symptoms and sexual dysfunction as compared to the symptoms observed in the women receiving TAM alone [13]. In the SOFT trial, hot flushes, sweating, decreased libido, vaginal dryness, insomnia, depression, musculoskeletal symptoms, hypertension, and glucose intolerance were reported more frequently in the 5-year OFS plus TAM group than in the TAM alone group, and the frequency of $\geq$ grade 3 adverse events was $31.3 \%$ in the OFS plus TAM group and $23.7 \%$ in the TAM alone group [6]. The SOFT trial also showed that the rate of non-adherence to OFS increased gradually with time, being $9.2,14.9,18.3$, and $21.9 \%$ at $1,2,3$, and 4 years, respectively. The estimated cost of OFS with goserelin or leuprorelin is about $\$ 2320$ per person-year. The balance 
Fig. 3 Disease-free survival in all patients. Kaplan-Meier curve of DFS in all patients $(n=215)$. The DFS rate at 6 years was 95.2 and $84.0 \%$ at 10 years

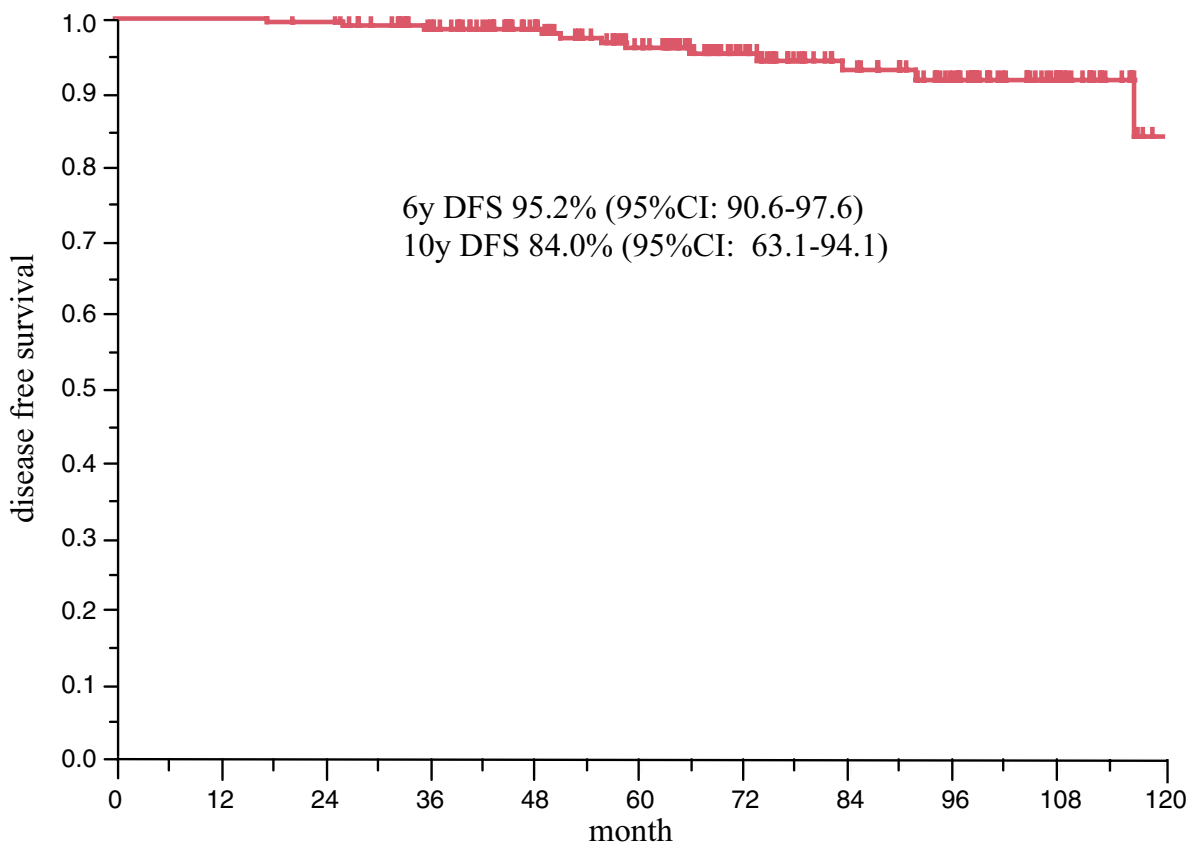

among the toxicity, cost, and clinical benefit should be carefully taken into consideration in relation to OFS as adjuvant therapy in premenopausal breast cancer patients.

\section{Study limitations}

This retrospective study was conducted without randomization in a small patient population at a single site. The number of events was relatively small. Most of the patients had low-risk disease with node-negative or no chemotherapy, which suggests the difficulty in evaluating the benefit of OFS. Premenopausal estradiol levels or menstruation after neo-adjuvant/adjuvant chemotherapy were not confirmed in the patients. Use of an aromatase inhibitor with OFS was not evaluated, because aromatase inhibitor agents are not yet approved for administration to premenopausal women in Japan.

Although adjuvant hormone therapy with 5-year OFS plus TAM is recommended for premenopausal patients with hormone receptor-positive breast cancer who are at a sufficient risk for recurrence to warrant adjuvant chemotherapy and who are expected to remain premenopausal until the end of the adjuvant chemotherapy, the optimal duration of OFS should be further investigated with a detailed assessment of the adverse events, QOL, financial burden with careful consideration given to the lack of definite evidence of a meaningful clinical benefit, i.e., impact, of this treatment on the disease outcome. Thus, our results should be confirmed in a prospective, randomized controlled trial including a larger patient population.

\section{Conclusions}

This study suggests that in premenopausal women with hormone receptor-positive breast cancer, the DFS of the patients did not differ significantly between patients receiving OFS for $\leq 3$ years and those receiving OFS for $>3$ years. A randomized trial is needed to establish the optimal duration of OFS for these patients.

Acknowledgements We thank the patients included in this trial, and the staff of Toranomon hospital. M.Uruma contributed to the data collection. K.Nonogaki, E.Ozeki, H.Sato, M.Takeshita, K.Igaki, and A.Yamamiya contributed to the data management. We thank Dr. Richard Theriault (University of Texas MD Anderson Cancer Center, Houston, TX) for his support and editorial assistance.

Author contributions The analyses were planned by YO, HK, and TT. The statistical analyses were performed by YO and KY. YT, NT, TO, $\mathrm{CK}$, and $\mathrm{YM}$ have made contribution of interpretation of data. All coauthors approved the final version.

Funding This work was supported by a research grant from Toranomon Hospital.

\section{Compliance with ethical standards}

Conflict of interest The authors have no competing interests to declare.

Ethical approval Approval for this retrospective study was obtained from the Ethics Committee of Toranomon Hospital.

Open Access This article is distributed under the terms of the Creative Commons Attribution 4.0 International License (http://creativecomm ons.org/licenses/by/4.0/), which permits unrestricted use, distribution, 
and reproduction in any medium, provided you give appropriate credit to the original author(s) and the source, provide a link to the Creative Commons license, and indicate if changes were made.

\section{References}

1. Fisher B, Dignam J, Bryant J, DeCillis A, Wickerham DL, Wolmark N, et al. Five versus more than five years of tamoxifen therapy for breast cancer patients with negative lymph nodes and estrogen receptor-positive tumors. J Natl Cancer Inst. 1996;88:1529-42.

2. Early Breast Cancer Trialists' Collaborative G. Effects of chemotherapy and hormonal therapy for early breast cancer on recurrence and 15-year survival: an overview of the randomised trials. Lancet. 2005;365:1687-717.

3. Fisher B, Jeong JH, Bryant J, Anderson S, Dignam J, Fisher ER, et al. Treatment of lymph-node-negative, oestrogen-receptorpositive breast cancer: long-term findings from National Surgical Adjuvant Breast and Bowel Project randomised clinical trials. Lancet. 2004;364:858-68.

4. Fowble BL, Schultz DJ, Overmoyer B, Solin LJ, Fox K, Jardines $\mathrm{L}$, et al. The influence of young age on outcome in early stage breast cancer. Int J Radiat Oncol Biol Phys. 1994;30:23-33.

5. Ahn SH, Son BH, Kim SW, Kim SI, Jeong J, Ko SS, et al. Poor outcome of hormone receptor-positive breast cancer at very young age is due to tamoxifen resistance: nationwide survival data in Korea-a report from the Korean Breast Cancer Society. J Clin Oncol. 2007;25:2360-8.

6. Francis PA, Regan MM, Fleming GF, Lang I, Ciruelos E, Bellet $\mathrm{M}$, et al. Adjuvant ovarian suppression in premenopausal breast cancer. N Engl J Med. 2015;372:436-46.

7. Karlsson P, Sun Z, Braun D, Price KN, Castiglione-Gertsch M, Rabaglio M, et al. Long-term results of International Breast
Cancer Study Group Trial VIII: adjuvant chemotherapy plus goserelin compared with either therapy alone for premenopausal patients with node-negative breast cancer. Ann Oncol. 2011;22:2216-26.

8. Arriagada R, Le MG, Spielmann M, Mauriac L, Bonneterre J, Namer M, et al. Randomized trial of adjuvant ovarian suppression in 926 premenopausal patients with early breast cancer treated with adjuvant chemotherapy. Ann Oncol. 2005;16:389-96.

9. Group LH-aiEBCO, Cuzick J, Ambroisine L, Davidson N, Jakesz $\mathrm{R}$, Kaufmann M, et al. Use of luteinising-hormone-releasing hormone agonists as adjuvant treatment in premenopausal patients with hormone-receptor-positive breast cancer: a meta-analysis of individual patient data from randomised adjuvant trials. Lancet. 2007;369:1711-23.

10. Burstein HJ, Lacchetti C, Griggs JJ. Adjuvant endocrine therapy for women with hormone receptor-positive breast cancer: american society of clinical oncology clinical practice guideline update on ovarian suppression summary. J Oncol Pract. 2016;12:390-3.

11. Shiba E, Yamashita H, Kurebayashi J, Noguchi S, Iwase H, Ohashi $\mathrm{Y}$, et al. A randomized controlled study evaluating safety and efficacy of leuprorelin acetate every-3-months depot for 2 versus 3 or more years with tamoxifen for 5 years as adjuvant treatment in premenopausal patients with endocrine-responsive breast cancer. Breast Cancer. 2016;23:499-509.

12. Compton CC, Byrd DR, Garcia-Aguilar J, Kurtzman SH, Olawaiye A, Washington MK. A Companion to the Seventh Editions of the AJCC Cancer Staging Manual and Handbook. AJCC Cancer Staging Atlas. New York, NY: Springer;2012.

13. Tevaarwerk AJ, Wang M, Zhao F, Fetting JH, Cella D, Wagner LI, et al. Phase III comparison of tamoxifen versus tamoxifen plus ovarian function suppression in premenopausal women with node-negative, hormone receptor-positive breast cancer (E-3193, INT-0142): a trial of the Eastern Cooperative Oncology Group. J Clin Oncol. 2014;32:3948-58. 